\title{
MENINGIOMA GROWTH DURING INTERFERON BETA-1A TREATMENT FOR MULTIPLE SCLEROSIS
}

\author{
Hugo Pereira Pinto Gama ${ }^{1,2}$, Antônio José da Rocha, , Carlos Jorge da Silva ${ }^{1,2}$, \\ Maria Fernanda Mendes ${ }^{3}$, José Carlos Esteves Veiga ${ }^{4}$, Carmen Lúcia Penteado Lancellotti ${ }^{5}$, \\ Victor Piana Andrade 6 , Charles Peter Tilbery ${ }^{3}$
}

Multiple sclerosis (MS) is the most common demyelinating disease of the central nervous system (CNS) with a long-term course in the majority of patients. As all chronic illnesses, it is possible to expect the concomitant occurrence of other diseases, like CNS tumors ${ }^{1,2}$.

Some studies have been described CNS tumors in MS patients and we were able to find 31 cases reported, including 6 meningiomas, in English literature ${ }^{1-3}$. Meningioma is rarely described in MS patients, particularly during treatment with Interferon beta (INFb).

We report meningioma growth in our MS patient during INFb-la treatment, depicted by brain MRI scans. Immu- nohistochemistry results are also confronted with those earlier reported.

\section{CASE}

A 51 year-old woman was diagnosed as having possible MS in 1992 due to numbness and weakness in her left lower limb. Fundoscopy and the remaining neurological examination were unremarkable at that time. In 1996, she had a second bout and was submitted to her first MRI that did not show evidence of the tumor. Cerebrospinal fluid (CSF) examination revealed oligoclonal bands and a diagnosis of definite MS was established. Serial MRI scans demonstrated several small demyelinating foci in the spi-
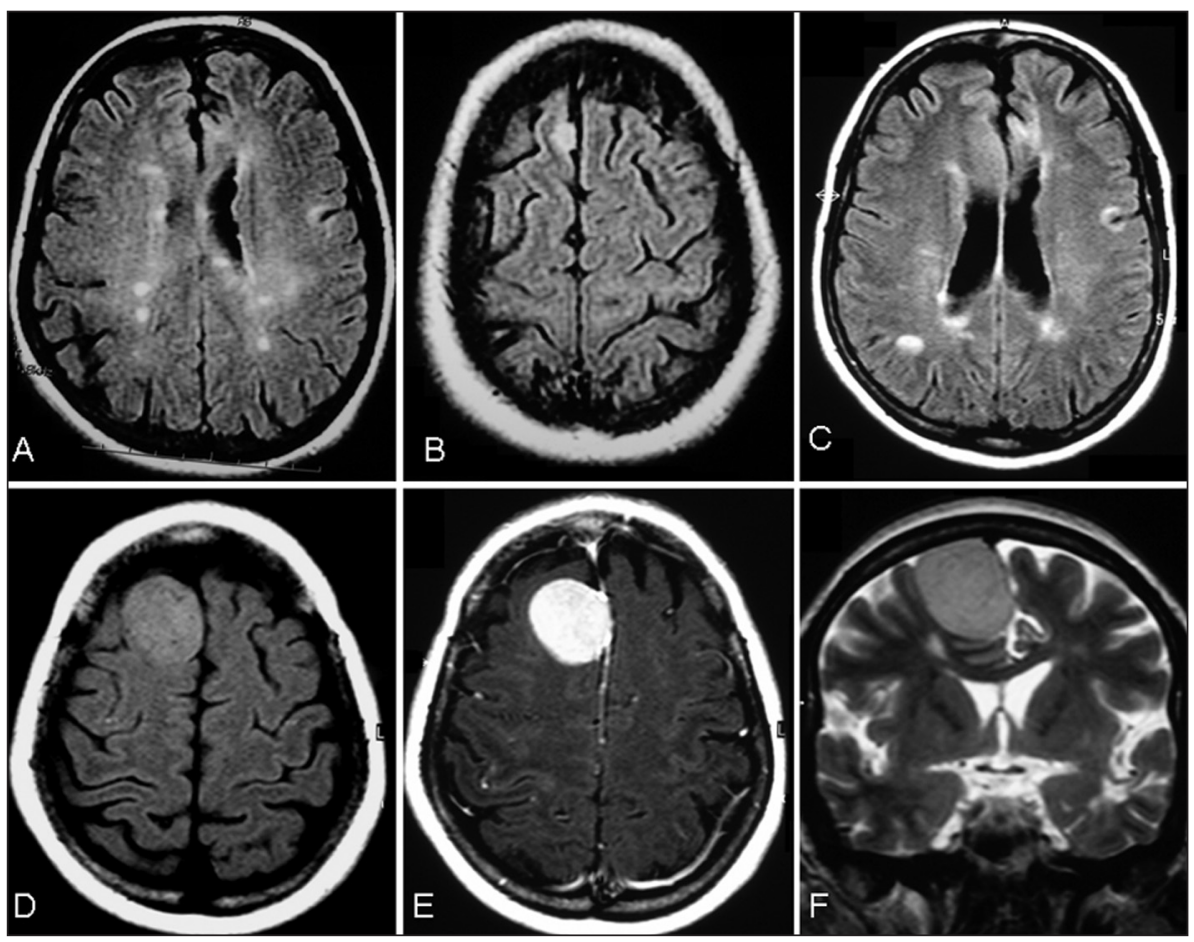

Fig 1. (A, B) Axial FLAIR (TR $11000 \mathrm{ms,}$ TE $140 \mathrm{~ms}$, IR $2600 \mathrm{~ms}$ ) images show multiple demyelinating foci in the white matter and a small isointense right frontal extra-axial mass. Followup (5 years later) examination (C-F). (C) Axial FLAIR image at the same level shows minimal increase of the demyelinating foci. (D) Axial FLAIR, (E) post-contrast T7-weighted (TR $509 \mathrm{~ms}$ TE14 ms), and (F) coronal T2-weighted (TR 5033 ms TE 100 ms) images reveal an evident meningioma enlargement (arrows).

\section{CRESCIMENTO DE MENINGEOMA DURANTE TRATAMENTO PARA ESCLEROSE MÚLTIPLA COM INTERFERON BETA-1A}

'Fleury - Diagnostic Center, Section of Radiology, São Paulo SP, Brazil; ${ }^{2}$ Santa Casa de Misericórdia de São Paulo, Section of Radiology, São Paulo SP - Brazil; 'Santa Casa de Misericórdia de São Paulo, Section of Neurology, São Paulo SP, Brazil; ' Santa Casa de Misericórdia de São Paulo, Section of Neurosurgery, São Paulo SP, Brazil; ${ }^{5}$ Santa Casa de Misericórdia de São Paulo, Section of Pathology, São Paulo SP, Brazil; ${ }^{6}$ Fleury - Diagnostic Center, Section of Pathology, São Paulo SP, Brazil

Received 7 December 2007, received in final form 28 February 2008. Accepted 18 March 2008.

Dr. Antônio José da Rocha - Rua Cincinato Braga 282 - 01333-910 São Paulo SP - Brasil. E-mail: antonio.rocha@fleury.com.br 

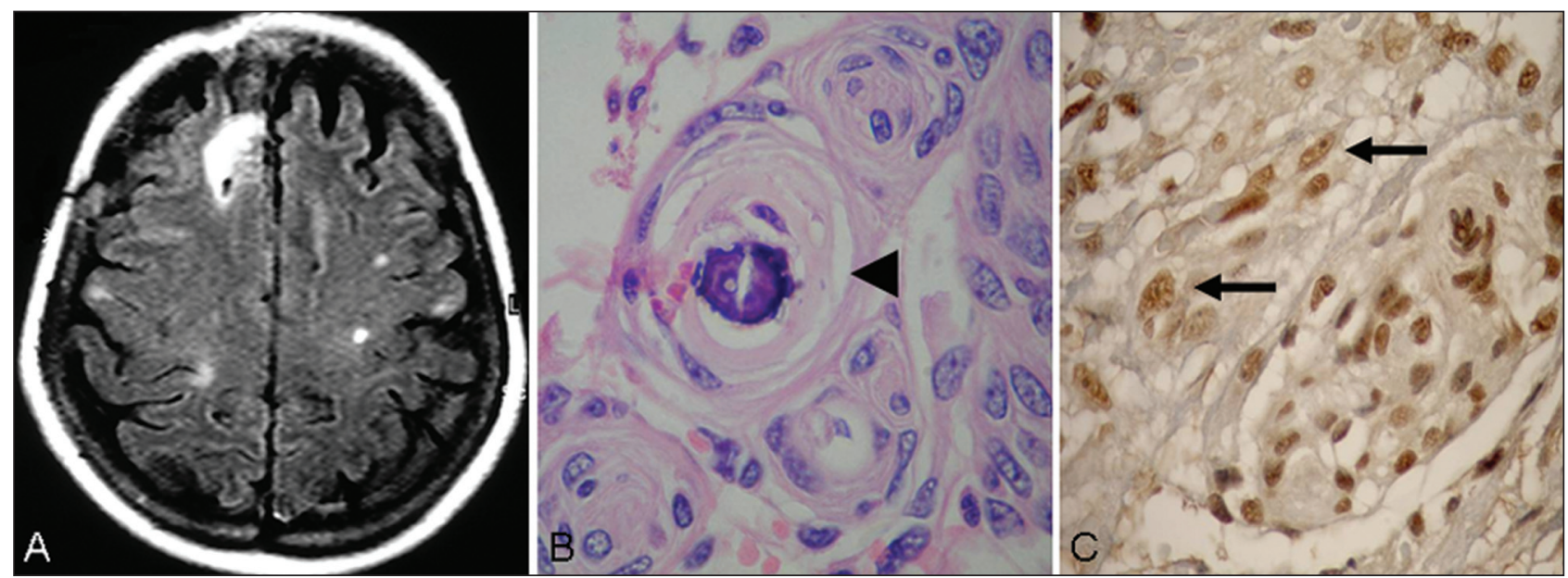

Fig 2. Postoperative follow-up. (A) Axial FLAIR image shows complete resection of the tumor with post-surgical signal abnormalities in the right frontal lobe. (B) Histological sections confirm a typical meningioma with proliferation of cells containing bland round oval nuclei in a mesenchymal collagenized matrix, with a tendency to form concentric whorls with psammoma bodies (arrowhead). (C) Immunohistochemistry section demonstrates a positive result for TGF-b receptor represented by cells showing a positive nuclear brown immunostaining (arrows).

nal cord, cerebral hemispheres, pons, and cerebellum associated with involvement of the callososeptal interface. In 1997, she started INFb-la (Rebif ${ }^{\oplus}$ ) therapy, $22 \mu$ g subcutaneously, 3 times a week. She presented only a few episodes of MS relapses since the beginning of this therapy.

In December 2000, we performed a follow-up MRI that revealed only a discrete increase in white matter lesions and a small well-circumscribed $(1.0 \mathrm{~cm}$ in diameter), extra-axial parafalcine tumor in the right frontal convexity, consistent with meningioma (Fig 1). There was no neurological deterioration at this time. Collateral effects like muscular pain and chills appeared in the fifth year of treatment. The drug was changed and she started to use another INFb-1a (Avonex ${ }^{\circledR}$ ), $30 \mu \mathrm{g}$ intramuscularly once a week, since 2002. After that, she remained stable with only 2 MS relapses until 2005.

Another follow-up MRI was performed in 2005, showing a considerable enlargement of the meningioma $(3.5 \mathrm{~cm}$ in diameter) and minimal increase in the number of demyelinating foci (Fig 1). The patient did not present neurological deficits related to the tumor.

In March 2006, she was submitted to a complete tumor surgical resection. Histological analysis confirmed the diagnosis of a typical meningioma. We performed immunohistochemistry analysis (Fig 2) that detected the presence of TGF-b receptor and showed negative results to all remaining receptors tested, including vimentin, platelet derived growth factor (PDGF), epithelial membrane antigen, estrogen, progesterone, and epidermal growth factor receptors. We did not change the MS treatment and the patient was bout-free after surgery until now.

The patient signed an inform consent for this publication.

\section{DISCUSSION}

Meningiomas are the most common primary non-glial intracranial tumor. There is a female predilection with a ratio of about 2:1 and they are also associated with neurofibromatosis type $2^{4}$. The higher incidence of brain tumors in MS patients is controversial ${ }^{1,2}$. Particularly, the concomitance of meningioma and MS has been reported, including both spinal and intracranial segments ${ }^{1-3}$.

Interferon alpha (INFa) is currently used for the treatment of unresectable or malignant meningiomas ${ }^{5}$ and this medication has 2 mechanisms of action: direct tumor cell inhibition ${ }^{6}$ and antiangiogenic activity ${ }^{7}$. INFb has been used in order to modify the natural course of MS, impairing the trafficking of inflammatory cells through the blood brain barrier ${ }^{8}$. However, its effect in meningiomas is not well understood.

Batay and Al-Merfy firstly described the possible relationship between meningioma and $\mathrm{MS}^{2}$, showing the progression of meningiomas during the treatment with INFb. They suggested that serious side effects of long-term INFb therapy, like autoimmune diseases and autoimmune aggression with lymphocytic infiltration, might be responsible for the occurrence and enlargement of meningiomas in MS patients.

Recently, Drevelegas et al. ${ }^{3}$ described a patient presenting enlargement of an intraventricular meningioma during INFb-1b treatment in a young woman with MS and suggested a possible relationship between the drug therapy and tumor growth. They argued that meningioma growth was related to positive PDGF and TGF-b receptors and the use of INFb.

In our case, we also observed a pronounced enlargement of a frontal convexity meningioma during INFb-1a treatment for MS 5 years after its detection in a follow-up 
MRI. However, instead of Drevelegas et al. ${ }^{3}$ results, our immunohistochemistry panel was negative to all receptors tested, except for TGF-b. This result is quite contradictory, because this particular receptor has an immunosuppressive activity ${ }^{9}$ and one study in vitro showed an inhibitory effect in meningioma cells ${ }^{10}$. Some authors demonstrated an upregulation of TGF-b in INFb treated patients, but this finding was not seen by others ${ }^{8}$. We agree that the role of TGF-b in this setting is controversial. Although INFa and INFb share some biological activities, the tumor enlargement observed by Drevelegas ${ }^{3}$ and us suggests that INFb do not share the same antitumoral properties of INFa ${ }^{5}$. This interesting suggestion, however, requires additional studies.

The mean annual meningioma growth rate in our patient $(0.5 \mathrm{~cm} /$ year) is similar to that previously reported". This growth rate does not exceed the normal range (0.24$1.0 \mathrm{~cm} /$ year) expected for meningiomas ${ }^{11,12}$.

Despite the limitations concerning this case report, our review of the literature let us to conclude that there are not enough arguments to confirm a definite relationship between meningioma growth, the presence of TGF-b receptor in the tumor cells and INFb therapy in our pa- tient. We believe the concomitance of these common conditions should be considered merely coincidental.

\section{REFERENCES}

1. Scully RE, Mark EJ, McNeely WF, Ebeling SH, Philips LD. Weekly clinicopathological exercises. Case 12. N Engl J Med 1997;336:1163-1171.

2. Batay F, Al-Merfy O. Growth dynamics of meningiomas in patients with multiple sclerosis treated with interferon: report in two cases. Acta Neurochir 2002;144:365-368.

3. Drevelegas A, Xinou E, Karacostas D, Parissis D, Karkavelas G, Milonas I. Meningioma growth and interferon beta- $1 \mathrm{~b}$ treated multiple sclerosis: coincidence or relationship? Neuroradiology 2005;47:516-519.

4. Atlas SW. Magnetic resonance imaging of the brain and spine. $3^{\text {rd }}$ edition. Lippincott Williams \& Wilkins. 2002:695-772.

5. Kaba SE, De Monte F, Bruner JM, et al. The treatment of recurrent unresectable and malignant meningiomas with interferon alpha-2b. Neurosurgery 1997;40:271-275

6. Magrassi L, De Fraja C, Conti L, et al. Expression of the JAK and STAT superfamilies in human meningiomas. J Neurosurg 1999;91:440-446.

7. Folkman J, Ingber D. Inhibition of angiogenesis. Semin Cancer Biol 1992;3:89-96.

8. Yong VW. Differential mechanisms of action of interferon-b and glatiramer acetate in MS. Neurology 2002;59:802-808.

9. Racke MK, Bonomo A, Scott DE. Cytokine-induced immune deviation as a therapy for inflammatory autoimmune disease. J Expert Med 1994;180:1961-1966.

10. Zhang ZJ, Muhr C, Wang JL. Interferon-alpha inhibits the DNA synthesis induced by PDGF and EGF in cultured meningioma cells. Anticancer Res 1996;16:1717-1723.

11. Herscovici Z, Rappaport Z, Sulkes J, Danaila L, Rubin G. Natural history of conservatively treated meningiomas. Neurology 2004;63:1133-1134.

12. Go RS, Taylor BV, Kimmel DW. The natural history of asymptomatic meningiomas in Olmsted County, Minnesota. Neurology 1998;51:1718-1720. 